\title{
Oscillatory $\mathrm{AAA}^{+}$ATPase Knk1 constitutes a novel morphogenetic pathway in fission yeast
}

\author{
Kathleen Scheffler ${ }^{a, b}$, Pierre Recouvreux ${ }^{c}$, Anne Paoletti ${ }^{a, b}$, and Phong T. Tran ${ }^{a, b, d, 1}$ \\ ${ }^{a}$ Centre de Recherche, Institut Curie, F-75248 Paris, France; ${ }^{b}$ CNRS-UMR144, F-75248 Paris, France; Institut de Biologie du Développement de Marseille, \\ CNRS-UMR7288, 13009 Marseille, France; and ${ }^{\mathrm{d} D e p a r t m e n t}$ of Cell and Developmental Biology, University of Pennsylvania, Philadelphia PA 19104
}

Edited by Thomas D. Pollard, Yale University, New Haven, CT, and approved November 3, 2014 (received for review April 19, 2014)

Cellular morphogenesis relies partly on cell polarization by the cytoskeleton. In the fission yeast Schizosaccharomyces pombe, it is well established that microtubules (MTs) deliver the spatial cue Tea1, a kelch repeat protein, to the tip regions to direct the growth machinery at the cell tips driving the linear extension of the rodshaped organism to maintain a straight long axis. Here, we report the characterization of Knk1 (kink), a previously unidentified member of the superfamily of ATPases associated with various cellular activities $\left(\mathrm{AAA}^{+}\right)$, whose deletion causes a unique morphological defect characterized by the formation of kinks close to cell tips. Through genetic analysis, we place Knk1 into a novel pathway controlling cell shape independently of MTs and Tea1. Knk1 localizes at cell tips. Its localization is mediated by the Knk1 $\mathrm{N}$ terminus and is enhanced upon ATP binding to the C-terminal ATPase domain. Furthermore, Knk1 tip recruitment is regulated by SRC-like adaptor 2 (Sla2) and cell division cycle 42 (Cdc42) independently of Sla2's role in endocytosis. Finally, we discovered that Knk1 shows an anticorrelated oscillatory behavior between the two cell tips at a periodicity that is different from the reported oscillatory Cdc42 dynamics.

oscillation | morphogenesis | kink

W ild-type fission yeast forms rod-shaped cells $4 \mu \mathrm{m}$ in diameter that grow linearly from the cell tips and divide medially. After division, growth resumes in a monopolar fashion before switching to a bipolar growth during NETO (new end take off) (1). Its morphogenesis is regulated by both, the microtubule (MT) and actin cytoskeletons, and defects in these respective cytoskeletons lead to characteristic shape changes. Mutants affecting actin-based structures usually partially fail to polarize growth at cell tips, resulting in plump cells; in contrast interfering with MT-related functions leads to the loss of a straight axis of growth, resulting in bent or T-shaped cells (Fig. $1 A$ ).

In interphase, actin forms two distinct structures: actin cables delivering, via myosin $\mathrm{V}$, secretory vesicles and actin patches representing sites of endocytosis (2). Briefly, the activity of the small GTPase cell division cycle 42 ( $\mathrm{Cdc} 42)$, constituting the core of the polarization machinery, is confined to the cell poles (3). Otherwise cells become roundish because of isotropic growth as observed upon inactivation of the NDR kinase Orb6 (4). Cdc42 activates formin For3, the formin responsible for actin cable nucleation (5). for $3 \Delta$ cells lacking actin cables partially lose their polarized state and become bulbous or swollen. Disruption in the endocytic pathway, such as deletion of the endocytic adaptor protein Sla2, results in a similar shape phenotype $(6,7)$.

MTs contribute to cell shape by marking cell tips as sites of growth through the deposition of a landmark protein complex, including the kelch repeat protein, Tea1 $(8,9)$. Interphase MTs organized in three to five bundles parallel to the cell's long axis are anchored to the nucleus with their minus ends. Their plus ends show dynamic behavior, extending toward cell poles and contacting the cortex for about 50-80 s. Simultaneously, the Tea1 protein complex is carried by MT plus ends in a plus tip microtubule aberrant length protein 3 (Mal3; EB1 homolog)dependent manner toward the cell tips, where Tea1, in turn, indirectly contributes to the activation of For3 (10). Upon loss of
Tea1, cells fail to maintain a straight axis and become bent (11). Similarly, an aberrant MT cytoskeleton leads to bent cells resulting from Tea1 complex mispositioning $(12,13)$. For example, the deletion of either Mal3, which results in very short MTs that do not reach cell ends $(14,15)$, or of the MT nucleator Mto1 (pericentrin homolog), which reduces the number of MT bundles, causes a bent shape (16).

In this study, we describe a novel gene, $k n k 1$ (kink, encoded by SPBC947.01/alf1), which plays a role in morphogenesis and founds a new class of shape mutants forming kinks. Knk1 belongs to the superfamily of ATPases associated with various cellular activities $\left(\mathrm{AAA}^{+}\right)$found in all organisms and implicated in various cellular processes such as protein degradation and membrane trafficking (17). Despite their diversity in function, they share a common core mechanism by assembling into ring-shaped hexamers. Interestingly, Knk1 shows a fascinating oscillatory behavior between the two cell tips.

\section{Results}

The knk1s Mutant Displays a Novel Kinked Shape Phenotype. We identified $k n k 1$ in a visual screen for new shape mutants using the genomewide library of haploid fission yeast deletion mutants (18). Intriguingly, cells carrying a deletion of $k n k l$ displayed a kink close to cell tips (Fig. $1 A$ and $B$ ). Kinks were observed, on average, in $8.2 \%$ of cells at $25^{\circ} \mathrm{C}$ and in up to $20.7 \%$ of cells after 3 h at $36{ }^{\circ} \mathrm{C}$ (Fig. $1 C$ ), usually at only one pole but rarely at both $\left(\sim 4 \%\right.$ of kinked cells at $\left.36^{\circ} \mathrm{C}\right)$. Although wild-type cells always displayed a straight long axis, kinked mutant cells were characterized by an angle $(\theta)$ between the tip growth axis and the otherwise straight long cell axis (Fig. $1 E$ ). The average angle of kinks was $25.6 \pm 7.7^{\circ}$ at $25^{\circ} \mathrm{C}($ Fig. $1 D)$.

\section{Significance}

We describe a novel pathway controlling fission yeast cell morphogenesis depending on Knk1, a member of the conserved ATPases associated with various cellular activities (AAA ${ }^{+}$) family regulating important cellular processes in all organisms and often functioning in unfolding, degradation, or dissociation of multimeric complexes. Deletion of $k n k 1$ (kink, encoded by SPBC947.01/ alf1) founds a new class of morphogenesis mutants exhibiting sudden changes in directional growth. Knk1 performs oscillations between the two cell ends at a periodicity different from the reported cell division cycle 42 oscillations. We conclude that at least two oscillatory systems may coexist in fission yeast to control cell morphogenesis. We speculate that oscillatory properties could represent a key feature of morphogenetic factors to control the growth machinery in a dynamic manner and achieve an accurate definition of cell shape.

Author contributions: K.S. designed research; K.S. performed research; P.R. contributed new reagents/analytic tools; K.S. and P.R. analyzed data; K.S., A.P., and P.T.T. wrote the paper

The authors declare no conflict of interest.

This article is a PNAS Direct Submission.

${ }^{1}$ To whom correspondence should be addressed. Email: phong.tran@curie.fr.

This article contains supporting information online at www.pnas.org/lookup/suppl/doi:10. 1073/pnas.1407226111/-/DCSupplemental. 


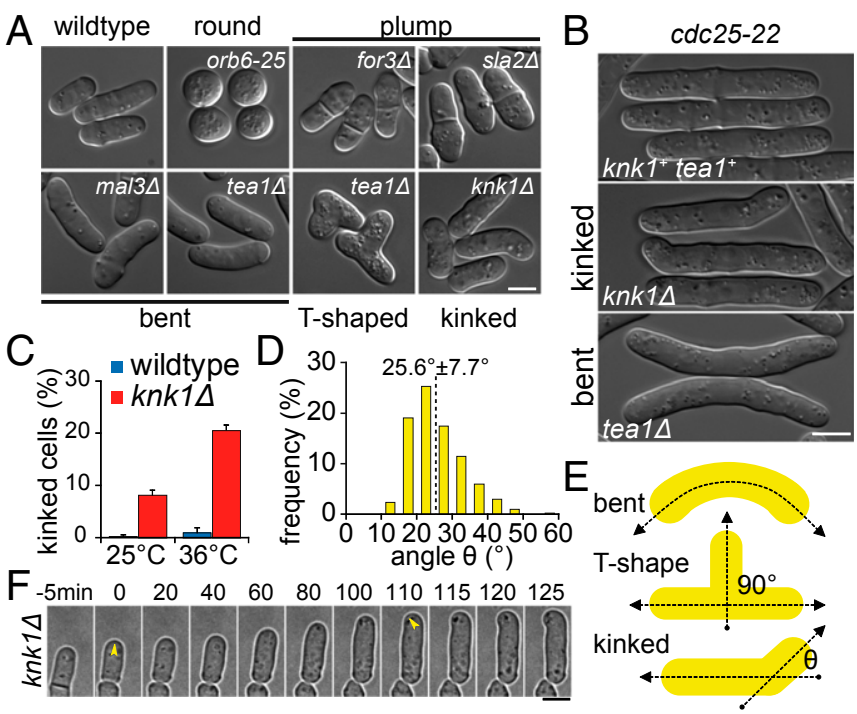

Fig. 1. knk1s has a novel kinked shape phenotype. (A) Differential interference contrast (DIC) images of shape mutants showing the normal rod shape of wild-type cells and the aberrant round (orb6-25, $7 \mathrm{~h}$ at $36{ }^{\circ} \mathrm{C}$ ), plump (for $3 \Delta$ and s/a2s), bent (mal3s and tea1 $\Delta$ ), T-shaped (tea1 $\Delta$ after starvation), and kinked ( $k n k 1 \Delta)$ shapes of morphology mutants (at $25{ }^{\circ} \mathrm{C}$ if not stated otherwise). (B) Kinked and bent cell morphology in cdc25-22 $k n k 1 \Delta$ and $c d c 25-22$ tea $1 \Delta$ strains after $3 \mathrm{~h}$ at $36^{\circ} \mathrm{C}$. (C) Percentage of wildtype and $k n k 1 \Delta$ cells displaying kinks at $25^{\circ} \mathrm{C}$ or after $3 \mathrm{~h}$ at $36{ }^{\circ} \mathrm{C}$. Bars represent means from three independent experiments $(n>300$ cells per experiment). Error bars indicate SD. (D) Distribution of angles $\theta$ in $k n k 1 \Delta$ cells after $3 \mathrm{~h}$ at $25^{\circ} \mathrm{C} ; n=261$. The dotted line indicates the mean angle of $25.6 \pm$ $7.7^{\circ}$. (E) Schematic highlighting the characteristics of a bent, T-shaped, or kinked morphology. $\theta$ is the angle between the growth direction (defined as the tip orientation) and the cell's long axis. $(F)$ Time-lapse images of a knk1 cells by brightfield microscopy at $36^{\circ} \mathrm{C}$. Arrowheads indicate the direction of growth. (Scale bars, $5 \mu \mathrm{m}$.)

To understand the origin of kinks, we followed their formation by time-lapse recording at $36^{\circ} \mathrm{C}$. $k n k 1 \Delta$ cells first grew linearly; then growth was reoriented toward another direction (Fig. $1 F$ ). After this event, the direction of tip growth remained straight. Kinks formed more frequently at old ends (70\% kinks, $n=60$ cells) at 5-150 min after cell separation and at a cell length of 7.6-13.6 $\mu \mathrm{m}$ but also could appear at new ends (30\% kinks), at 30-105 min after cell separation and at a cell length of 8.4-12.7 $\mu \mathrm{m}($ Fig. S1B). In a few kinked cells $(6 \%)$, kinks formed at both cell tips; this event could be observed more frequently $(34 \%)$ in a $c d c 25-22$ background at $36{ }^{\circ} \mathrm{C}$ in which the growth phase is extended (Fig. S1 $A$ ). Taken together, these results suggest that kinks are not produced when growth resumes at the old end after sister cell separation nor at the new end after NETO but arise at low frequency at any time point of the growth phase.

Overexpression of Knk1-YFP in knkls cells led to a gradual disappearance of kinks, demonstrating that Knk1 expression was not able to correct established kinks but did prevent the formation of new kinks (Fig. S1C). Old kinks were lost after several rounds of division.

Importantly, the kinked phenotype clearly differed from the bent phenotype, which is characterized by a gradual directional deflection of growth resulting in a curvature (Fig. $1 D$ and Fig. $\mathrm{S} 1 D$ ) evidenced in a $c d c 25-22$ background (Fig. $1 B$ ). Bent cells frequently are observed in teals cells $(18.2 \%)$ or MT-defective mutants such as mal3$\Delta(19.4 \%)$ (Fig. $2 A$ and $B$ ) in which Tea1 is dispersed along the cortex as shown for tip1 (12), both resulting in mispositioning of the growth machinery away from cell tips. Indeed, the majority of tea $1 \Delta$ and mal3s cells with morphological defects displayed a gradual directional deflection of growth. Kinked cells could be observed only at low frequency $(2.7 \%$ or $3.7 \%)$ in these mutants, as could T-shapes $(0.5 \%$ or $0 \%)$ (Fig. 2
$A$ and $B$ ) which exhibit a medial outgrowth approximately perpendicular to the main long axis (Fig. $1 D$ and Fig. $\mathrm{S} 1 E$ ). Thus, the teals and MT mutants differ significantly from the knkls mutant that displays a very low frequency $(2 \%)$ of bent cells and a complete absence of T-shapes (Fig. $2 A$ and $B$ ). In addition, unlike Tea1, Knk1 is not necessary for the initiation of bipolar growth during NETO (Fig. S1F) (19).

We conclude that the kink phenotype defines a novel class of shape mutants in fission yeast.

Aggravation of Kinked Phenotype by MT Defects. The new shape phenotype of the $k n k 1 \Delta$ mutant suggested that Knk1 may control cell morphogenesis by orientating growth direction independently of the MT/Tea1 pathway. Accordingly, the MT cytoskeleton labeled with GFP-Atb2 (alpha tubulin) appeared indistinguishable from the wild-type MT cytoskeleton in straight or kinked knk1s cells (Fig. S2A). We observed no significant changes in MT dynamics (Fig. $2 C$ ), demonstrating that Knk1 is not involved in MT regulation and that the kinks present in knkls cells are not a consequence of an abnormal MT behavior.
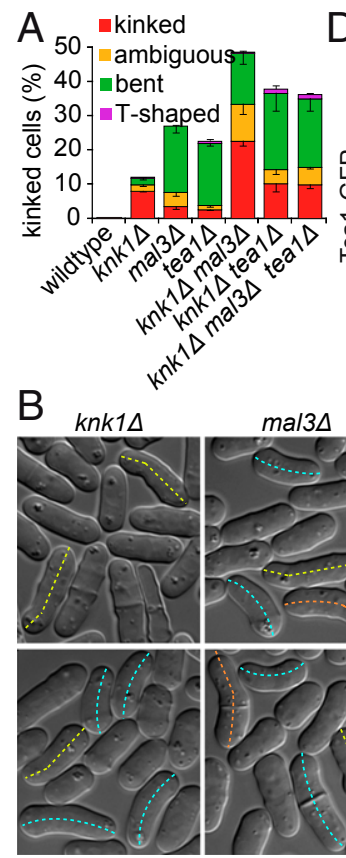

tea1ム mal34

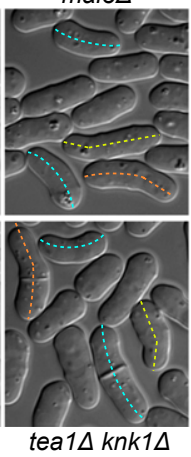

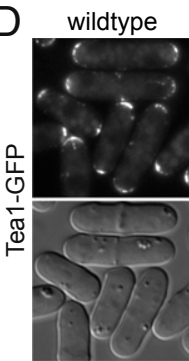

mal3 $\Delta k n k 1 \Delta$

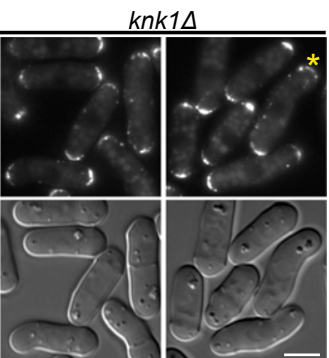

E

\begin{tabular}{|c|c|c|c|c|c|}
\hline strain & $\begin{array}{c}\text { Growth Rate } \\
(\mu \mathrm{m} / \mathrm{min})\end{array}$ & $\begin{array}{c}\text { Shrinkage } \\
\text { Rate }(\mu \mathrm{m} / \mathrm{min})\end{array}$ & $\begin{array}{c}\text { Catastrophe } \\
(\text { events/min) }\end{array}$ & $\begin{array}{c}\text { Rescue } \\
(\mathrm{events} / \mathrm{min})\end{array}$ & $\begin{array}{c}\text { Dwell Time } \\
(\mathrm{s})\end{array}$ \\
\hline wildtype & $2.27 \pm 0.58$ & $8.25 \pm 2.31$ & $0.35 \pm 0.14$ & $0.84 \pm 0.65$ & $68.8 \pm 35.8$ \\
\hline knk1 1 & $2.34 \pm 0.68$ & $8.78 \pm 2.81$ & $0.35 \pm 0.13$ & $0.73 \pm 0.47$ & $63.5 \pm 37.0$ \\
\hline p-Value & 0.55 & 0.3 & 0.82 & 0.18 & 0.43 \\
\hline
\end{tabular}

Fig. 2. Knk1 supports straight growth independently of MTs and Tea1. (A) Percentage of kinked, bent, and T-shaped cells and cells with ambiguous "kink-like" morphology, including cells with angles close to the middle of the cell and cells in which one half is bent and one half is kinked, in wild-type and mutant cells at $25^{\circ} \mathrm{C}$. Bars represent means from three independent experiments ( $n>300$ cells per experiment). Error bars indicate SD. (B) DIC images of the mutants shown in $A$. Dotted lines indicate midlong axis of cells with $a b-$ normal shapes. Yellow represents kinked cells, blue represents bent cells, and orange represents ambiguous cells. (C) MT dynamic parameters in wild-type and $k n k 1 \Delta$ cells (mean \pm SD; $n=50$ for each value). (D) Tea1-GFP localization in wild-type and $k n k 1 \Delta$ cells. The yellow asterisk marks a kinked tip with asymmetrical distribution of Tea1. (E) Mean angles at $25^{\circ} \mathrm{C}: k n k 1 \Delta\left(25.6 \pm 7.7^{\circ}\right)$ $k n k 1 \Delta$ tea $1 \Delta\left(27.3 \pm 9.8^{\circ}, P=0.01\right), k n k 1 \Delta$ mal3s $\left(38.3 \pm 13.6^{\circ}, P<10^{-37}\right)$, and $k n k 1 \Delta$ mal3s tea $1 \Delta\left(31.4 \pm 11.8^{\circ}, P<10^{-8}\right) . n \geq 197$. (Scale bars, $5 \mu \mathrm{m}$.) 
We thus tested if $k n k 1$ deletion may be synthetic with MTdefective mutants. A double knk1 $\Delta$ mal3s mutant indeed showed a dramatic increase in the frequency of kinked cells to $22.9 \%$ or even to $33.8 \%$ if ambiguous kink-like phenotypes (e.g., cells with an angle close to the cell middle) are included (Fig. $2 A$ and $B$ ). Additionally, the kink angle was enhanced from $25.6^{\circ}$ in $k n k 1 \Delta$ to $38.3^{\circ}$ in this background (Fig. $2 E$ and Fig. S2B). These results suggested that kinks could be produced by transient mispositioning of Tea1, resulting in an abrupt reorientation of growth. If so, the absence of Tea1 should suppress the kinked phenotype of $k n k 1 \Delta$ cells. However, in a double knkls teals mutant the frequency $(10.2 \%)$ and angle $\left(27.3^{\circ}\right)$ of kinks were similar to those in a knk1 1 single mutant (Fig. $2 A, B$, and $E$ ). This double mutant also had the same percentage $(22.3 \%)$ of bent cells as the teals single mutant. Thus, Tea1 is not required for the formation of kinks in knk1s cells, and Knk1 and Tea1 function independently of one another. Consistently, Tea1-GFP localized symmetrically around most cell tips in the knkls mutant (Fig. 2D), although Tea1 was distributed asymmetrically around some kinked tips (Fig. 2D, yellow asterisk). One possible reason for this asymmetric localization is that MTs may not always reach the center of a kinked tip. Strikingly, a knk1s mal3s teals triple mutant exhibited $10.1 \%$ kinked cells, as compared with more than $20 \%$

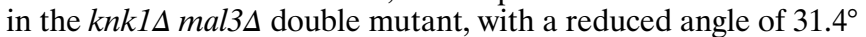
as compared with $38.3^{\circ}$ in $k n k 1 \Delta$ mal3s double mutant (Fig. $2 A$, $B$, and $E$ ). This observation shows that in the knk1 $\Delta$ background Tea1 absence suppresses the aggravating effect of mal3 $\Delta$. Similar results were obtained with other MT mutants, such as $m t o 1 \Delta$, in which Tea1 localization is affected (13), but not with mutants with more stable MTs in which Tea1 distribution presumably is more normal (Fig. S2 $C-E$ ) (20).

Taken together, our data suggest, that unlike MTs, Knk1 does not contribute to Tea1 localization. Knk1 appears to constitute a novel pathway supporting linear growth in an MT- and Tea1independent manner.

Knk1 Localizes to Cell Tips. To understand Knk1 function, we analyzed its intracellular distribution by expressing Knk1 tagged with two copies of GFP from its endogenous locus under control of its own promoter. The fusion protein was functional, because cells expressing Knk1-2xGFP were morphologically indistinguishable from wild-type cells.

Knk1 showed a cell cycle-dependent localization pattern (Fig $3 A$ ). In interphase, Knk1 was detected at one tip in short preNETO cells and at one (25\%) or both (75\%) tips in longer postNETO cells. During mitosis, concomitant with the formation of the acto-myosin cytokinetic ring, Knk1 dissociated from cell tips and appeared as patches at the medial cortex before condensing into a ring that constricted as cell division proceeded. Remarkably, the contractile ring constituted by Knk1 resided immediately outside the acto-myosin ring (Fig. S $3 A$ ), as described for $\mathrm{Cdc} 42$ guanine nucleotide exchange factors (GEFs) (21). Knk1 dissociated from the cortex upon cell separation and reappeared subsequently at old cell ends.

Knk1 Tip Localization Is Mediated by its $\mathbf{N}$ Terminus and Enhanced by the ATPase Domain. Knk1 belongs to the $\mathrm{AAA}^{+}$superfamily of ATPases that typically assemble into ring-shaped hexamers through interactions between ATPase domains (22). This protein family shows high conservation within the ATPase domain located at the $\mathrm{C}$ terminus but is quite divergent in other regions. Accordingly, based on sequence analysis, Knk1 was similar to $\mathrm{AAA}^{+}$ATPases from fungi to human, including the human MTsevering enzymes fidgetin and spastin (Fig. 3B). Knk1 also contains a putative C-terminal helix found in the endocytic trafficking-related vacuolar protein sorting $4(\mathrm{Vps} 4)$ protein, but its function remains unclear (23). In contrast, the $\mathrm{N}$ terminus of Knk1 exhibited no resemblance to other proteins.

First, to test how the $\mathrm{N}$ and $\mathrm{C}$ termini contributed to Knk1 function and localization, we expressed truncated versions of Knk1 fused to 2xGFP from the knk1 endogenous locus in
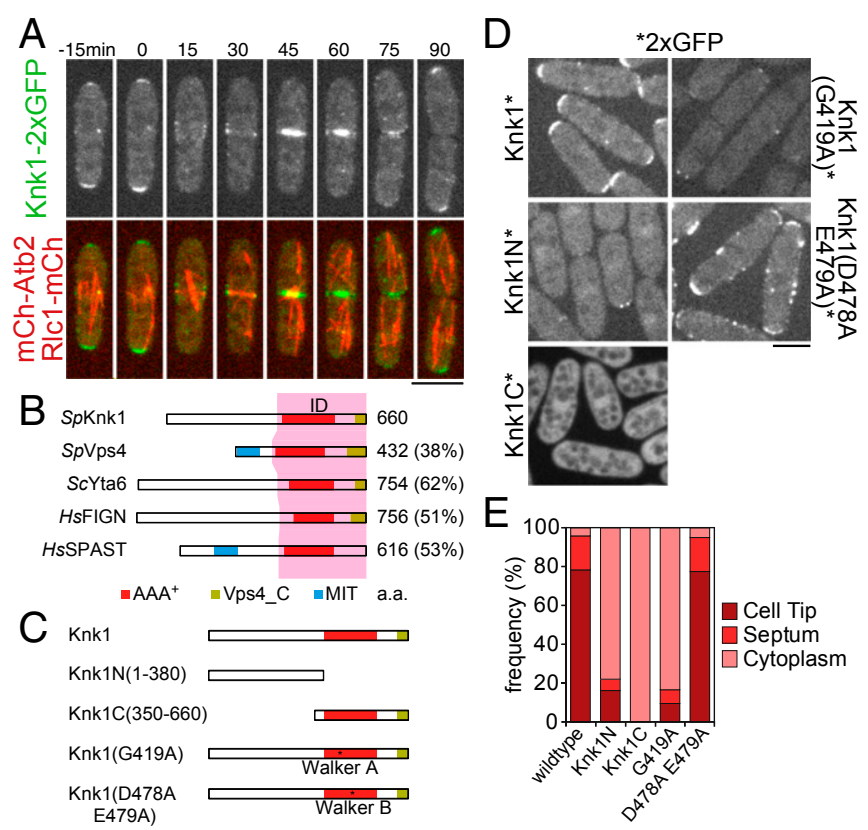

Fig. 3. Knk1N mediates tip binding that is enhanced by the ATPase domain. (A) Time-lapse images of Knk1-2xGFP expressed from the endogenous promoter in a strain coexpressing mCherry-Atb2 (tubulin) and regulatory light chain 1 (RIc1)-mCherry (acto-myosin ring) through the cell cycle at $25^{\circ} \mathrm{C}$. Knk1-2xGFP is presented as single medial z-section, and mCherry-Atb2 RIc1mCherry is presented as maximum projection of 3D stacks. $(B)$ Schematic representation of homology between $\mathrm{Knk} 1$ and related $\mathrm{AAA}^{+}$proteins. Protein length is expressed in amino acids. The region with high similarity (limited to the $\mathrm{AAA}^{+}$ATPase domain) is highlighted, and percent identity is shown. Other domains depicted are the MT interacting-trafficking domain (MIT) and the Vps4 oligomerization domain (Vps4_C). (C) Schematic of truncated and mutated constructs of Knk1 expressed from the knk1 endogenous locus and tagged with 2xGFP. (D) Localization of Knk1 constructs at $25^{\circ} \mathrm{C}$. Image of $\mathrm{Knk} 1 \mathrm{C}$ localization was scaled differently because of increased protein levels. Asterisks indicate Knk1 = 2xGFP. (E) Subcellular distribution of wild-type and Knk1 constructs; $n=300$. Bars correspond to percentage of cells showing a distinct signal at cell tips or septum and without a specific cortical signal (cytoplasm). (Scale bars, $5 \mu \mathrm{m}$.)

replacement of full-length Knk1 (Fig. 3C). Protein levels for Knk1N were similar to those of wild type and increased sixfold for Knk1C (Fig. S3C). Cells expressing Knk1N or Knk1C phenocopied $k n k 1$ deletion (Fig. S3B), demonstrating that both regions are required for Knk1 function. Knk1C was exclusively cytoplasmic, whereas Knk1N was weakly localized to the cell tips or septum region in $\sim 20 \%$ of cells (Fig. $3 D$ and $E$ ), indicating that tip recruitment is mediated by the $\mathrm{N}$ terminus but that recruitment efficiency might be enhanced by the ATPase domain, presumably by formation of hexameric complexes.

To test this hypothesis, we created mutant versions of Knk1 by site-directed mutagenesis in the two well-conserved Walker motifs of the ATPase domain (Fig. 3C) (24). The Walker A motif constitutes the ATP-binding site. Mutating this site abolishes ATP binding and often leads to dissociation of the hexameric complexes, whereas the Walker B motif allows ATP binding and hexameric assembly but inhibits ATP hydrolysis. Protein levels of these mutants were comparable to those in wild-type Knk1 (Fig. $\mathrm{S} 3 C$ ). Both mutant $k n k 1$ alleles produced kinked cells (Fig. S3B), demonstrating that a fully active ATPase is required for correct cell morphogenesis. Consistent with our hypothesis that Knk1 oligomerization enhances tip binding of the $\mathrm{N}$ terminus, Knk1 (G419A) mutated in the Walker A motif showed a weak tip signal similar to that in Knk1N (Fig. $3 D$ and $E$ ). In contrast, the Walker B mutant, Knk1(D478A E479A), was recruited to cell tips, as was 
wild-type Knk1 (Fig. $3 D$ and $E$ ), implying that ATP hydrolysis is not required for Knk1 localization.

Taken together, these data indicate that Knk1 acts as an ATPase at the cell tip. Furthermore, recruitment to the tip is controlled by the $\mathrm{N}$ terminus and is enhanced upon ATP binding, possibly through the formation of Knk1 hexamers, which may allow the cooperation of several $\mathrm{N}$ termini.

Knk1 Localization at the Cell Tip Is Independent of MTs and Actin Cables. Next, we examined whether Knk1 recruitment to cell tips is dependent on the actin or MT cytoskeleton. When cells were treated with $50 \mu \mathrm{g} / \mathrm{mL}$ carbendazim (MBC) to depolymerize MTs, Knk1 localization was not affected (Fig. $4 A$ ). Knk1 also stained growing cell tips in mutants of the MT-Tea1 pathway, mal3s or tea1 $\Delta$, and even after $4 \mathrm{~h} \mathrm{MBC}$ treatment (Fig. S4A).

In contrast, Knk1 was no longer detected at cell tips upon treatment with $100 \mu \mathrm{M}$ latrunculin A (LatA), an inhibitor of F-actin (Fig. $4 B$ ), leading to disassembly of F-actin patches marked by coronin-like protein 1 (Crn1)-GFP (Fig. $4 B$ ). Interestingly, although Crn1 patches were restored 20 min after washout of LatA, Knk1 tip localization was restored only after $90 \mathrm{~min}$, suggesting that Knk1 localization was not controlled simply by the presence of F-actin but rather by F-actin-based processes that are required for the establishment and maintenance of Knk1 at cell tips. Alternatively, Knk1 degradation might be induced by LatA, resulting in long recovery periods after drug washout.

Importantly, Knk1 levels at cell tips in a for $3 \Delta$ mutant that lacks F-actin cables were comparable to levels in wild-type cells (Fig. $4 C$ and $E$ ) (25), demonstrating that Knk1 tip localization relies on F-actin patches rather than on F-actin cables.

Sla2 and Cdc42 Regulate Knk1 Localization. Because F-actin patches correspond to sites of endocytosis, we next tested the impact of

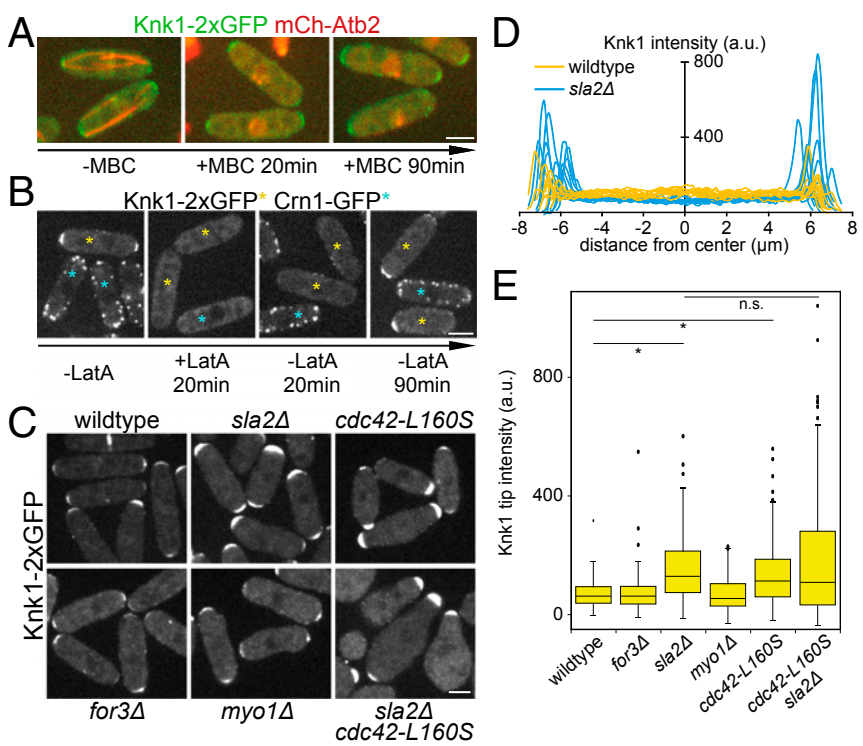

Fig. 4. Sla 2 and $C d c 42$ regulate Knk1 cell tip localization. ( $A$ and $B$ ) Cells expressing Knk1-2xGFP mCherry-Atb2 $(A)$ or Knk1-2xGFP (yellow asterisks) or Crn1-GFP (actin patch, blue asterisks) $(B)$ were treated with $50 \mu \mathrm{g} / \mathrm{mL} \mathrm{MBC}(A)$ or $100 \mu \mathrm{M}$ LatA $(B)$ for $20 \mathrm{~min}$. Drug was washed out, and images recorded at the indicated time points. $(C) \mathrm{Knk} 1$ localization in wild-type and mutant strains. $(D)$ Line scan of Knk1-2xGFP fluorescence intensity along the long axis of 12 individual wild-type and sla2 $\Delta$ cells of comparable length. Intensities were measured over the full cell width at each point along the cell axis. a.u., arbitrary units. (E) Knk1 intensity (a.u.) averaged over individual tips in wild-type cells (70.4 \pm 46.9 a.u.) and in the mutants for $3 \Delta(73.9 \pm 70.3, P=0.68)$, sla $2 \Delta(150.3 \pm$ $\left.119.9,{ }^{*} P<10^{-8}\right)$, myo1s $(71.6 \pm 59.5, P=0.87)$, cdc42-160S $(142.4 \pm 119.9$, $\left.{ }^{*} P<10^{-6}\right)$, and sla2 2 cdc24-L160S $\left(197.7 \pm 225.3, P<10^{-6}\right.$ relative to wild type, $P=0.07$ relative to sla2 $\Delta$ ). $n=100$. (Scale bars, $5 \mu \mathrm{m}$.) n.s., not significant. endocytosis defects on Knk1 distribution. In the sla $2 \Delta$ mutant, in which endocytosis is blocked before membrane ingression by the uncoupling of membrane and actin dynamics (26), Knk1 levels at cell tips were two- to threefold higher than in wild-type cells (Fig. $4 C$ and $E$ ), but global protein levels were not altered, as shown by Western blot (Fig. S4E). Line scans along the long axis confirmed increased tip but lowered cytoplasmic levels (Fig. 4D), demonstrating that Knk1 was more bound to cell tips.

The observed accumulation at tips in the sla $2 \Delta$ mutant suggested that Knk1 may be internalized by endocytosis. We therefore tested Knk1 distribution in two other endocytic mutants, class I myosin (myols) and Wasp homolog (wsp1s) mutants, which fail to activate F-actin nucleation by Arp2/3 (27-29) and lead to the accumulation of Sla2 at cell tips (Fig. S4D). Surprisingly, Knk1 tip levels were not increased significantly in either mutant (Fig. $4 C$ and $E$ and Fig. S4C), demonstrating that Knk1 accumulation in sla $2 \Delta$ cells is not caused by endocytosis defects. Consistently, costaining of Sla2-TagRFP and Knk1-2xGFP revealed only a partial overlap (Fig. S $4 F$ ). As demonstrated by a line scan along the cell tip (asterisk in Fig. S4F), Sla2 formed patches at the tip that moved inwards (arrow in Fig. S4F), but Knk1 stained tips more uniformly and never was detected in patches distant from the cell tip cortex. This observation suggests Knk1 is not directly associated with F-actin patches, although we cannot exclude the possibility that there may be a minor pool of Knk1 molecules at endocytic sites.

We next wondered whether Knk1 might function in endocytosis, which can be monitored by the uptake of the lipophilic dye FM4-64. The control sla2s cells showed a significant disruption in FM4-64 uptake, but the knk1s cells did not (Fig. S4G). Thus, $\mathrm{Knk} 1$ is not required for proper endocytosis.

As mentioned above, during cell division Knk1 forms a contractile ring similar to that formed by Cdc42 GEFs (21). We therefore wondered whether Cdc42 activity might impact Knk1 distribution. To test this notion, we used the temperature-sensitive allele $c d c 42-L 160 S$ (30-32) that exhibits no defects in the early steps of endocytosis but reduced intensity of active Cdc42 at cell tips monitored by $\mathrm{Cdc} 42 / \mathrm{Rac}$ interactive binding (CRIB)GFP (33) at $25^{\circ} \mathrm{C}$. Intriguingly, Knk1 accumulated to high levels at tips in these conditions (Fig. $4 C$ and $E$ ), similar to sla $2 \Delta$. However, Sla2 localized to actin patches in $c d c 42-L 160 S$ cells (Fig. S4D), demonstrating that increased Knk1 tip intensity was not caused simply by the absence of Sla2. Furthermore, a double sla2s cdc42-L160S mutant did not differ significantly from a single sla $2 \Delta$ mutant (Fig. $4 C$ and $E$ ), indicating that Sla2 and $\mathrm{Cdc} 42$ function in the same pathway to regulate Knk1 recruitment to cell tips in an endocytosis-independent manner.

Knk1 Exhibits Novel Oscillatory Dynamics at Cell Tips. When we visualized Knk1-2xGFP in sla2s cells at 1-min intervals, we observed that Knk1 was highly dynamic at cell tips, continuously undergoing cycles of binding and detachment (Fig. $5 A$ and $B$ ). Strikingly, the behavior of the two cell tips appeared anticorrelated, and this observation was confirmed using a cross-correlation function (cross-correlation coefficient $r=-0.39 \pm 0.24$ ) Furthermore, in $\sim 60 \%$ of cells, these oscillations appeared to be periodic with an average periodicity of $13.9 \mathrm{~min}$ (frequency = $0.072 \cdot \mathrm{min}^{-1}$ ) obtained by Fast Fourier Transform (FFT) analysis on all cells and all recorded intervals, whether or not the oscillatory behavior was detected by eye (Fig. 5C). A similar periodicity of 13.5 min was detected when FFT was performed for a subgroup of cells exhibiting oscillations noticeable by eye (Fig. S5A), demonstrating the robustness of the FFT analysis.

When we followed tip intensities of Knk1-2xGFP in wild-type cells, no striking oscillatory behavior could be observed by eye (Fig. S5 $B$ and $C$ ), nor was a specific frequency detected by FFT analysis (Fig. S5D). Nevertheless, Knk1 often showed anticorrelated fluctuations (marked by arrows in Fig. S5C) with a cross-correlation coefficient $r=-0.16 \pm 0.25$, in contrast to uncorrelated fluctuations seen for Tea1-GFP with $r=0.04 \pm$ 0.34 . This result indicates that the two cell tips compete for Knk1 

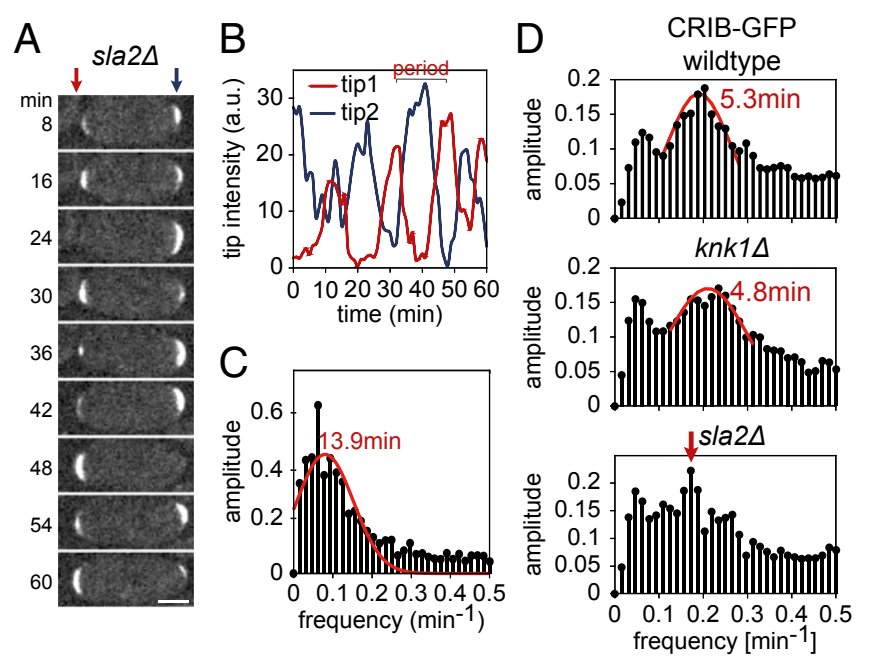

Fig. 5. Oscillations and fluctuations of Knk1-2xGFP at cell tips. (A) Timelapse images of Knk1-2xGFP in an sla2 $\Delta$ strain at $25^{\circ} \mathrm{C}$. (Scale bars, $5 \mu \mathrm{m}$.) $(B)$ Knk1-2xGFP tip intensities at 1-min intervals in the sla2 $\Delta$ cell shown in $A$. The period is defined as the average time span between two subsequent maxima of intensity (amplitude). Periodicity $=1 /$ frequency. $(C)$ Frequency spectrum of Knk1-2xGFP tip intensities analyzed by FFT analysis using all recorded intervals in sla2 $\Delta ; n=25$. The red line represents a Gaussian function of intervals of frequencies with higher amplitudes indicating average frequencies of $0.072 \cdot \mathrm{min}^{-1}$ for sla2s. (D) Frequency spectrum of CRIB-GFP in selected intervals (with periodic anticorrelated dynamics detected by eye) in wild-type cells $\left(n=14 ; 0.19 \cdot \mathrm{min}^{-1}\right)$ and in the mutants $k n k 1 \Delta(n=13$ $\left.0.21 \cdot \mathrm{min}^{-1}\right)$ and $\operatorname{sla} 2 \Delta[n=8$; not determined; the arrow indicates potential frequency at $0.18 \cdot \mathrm{min}^{-1}(5.6 \mathrm{~min}$ periodicity)].

and that the detachment of Knk1 from one tip allows the accumulation of Knk1 at the second competing tip. Because we failed to detect an oscillatory behavior for Knk1 in wild-type cells, the periodic oscillations might be triggered by the deletion or inactivation of sla 2 and $c d c 42$. Alternatively, Knk1 also might undergo a periodic oscillation in wild-type cells, but the dynamics of tip-bound Knk1 might not be measured reliably enough to detect them because of the strong fluctuations in cytoplasmic intensity (Fig. S5E).

A recent study using CRIB-GFP as a reporter for active $\mathrm{Cdc} 42$ evidenced anticorrelated oscillations of $\mathrm{Cdc} 42$ at cell tips with an average period of 5 min (34). To determine whether Knk1 and Cdc42 dynamics were linked, we analyzed the dynamics of Knk1 in the $c d c 42-L 160 S$ mutant. Knk1 oscillated with a period of 14.8 min in an anticorrelated fashion $(r=-0.3 \pm 0.31)$ (Fig. S5 $F-H)$, similar to oscillations seen in sla2s. CRIB-GFP dynamics could not be determined in this mutant, because levels were dramatically decreased (30). However, the detection of Knk1 oscillation despite a reduction of active $\mathrm{Cdc} 42$ in cell tips strongly suggests that Knk1 oscillations are not driven by Cdc42 dynamics. Conversely, we also tested whether Cdc42 oscillations were influenced by the deletion of knkl. In wild-type cells, CRIB-GFP oscillations had an average periodicity at $5.3 \mathrm{~min}$ (Fig. $5 D$ ), consistent with a previous report (34). CRIB-GFP distribution and oscillation were unaffected in $k n k 1 \Delta$ cells with an average period of $4.8 \mathrm{~min}$ (Fig. $5 D$ and Fig. S5I). Finally, we studied CRIB dynamics in sla2 $\Delta$ cells (Fig. S5I), in which Knk1 exhibited anticorrelated oscillations at 13.9-min intervals. Although less clearly than in wild-type cells, we observed a peak at a frequency of $0.18 \cdot \mathrm{min}^{-1}$ (5.6 $\mathrm{min}$ ), which is similar to observations in wildtype and knk1s cells (Fig. 5D), implying that Knk1 and Cdc42 oscillate at different frequencies in sla $2 \Delta$.

Finally, to determine if the Knk1 oscillation is linked to its turnover at cell tips, we performed fluorescence recovery after photobleaching (FRAP) experiments of cell tips. Knk1 intensity recovered partially, about $30 \%$ in wild-type and $15 \%$ in sla $2 \Delta$ cells, with a half-time $\left(\tau_{1 / 2}\right)$ of $\sim 6 \mathrm{~s}$ for both (Fig. S5J), suggesting that a large fraction of Knk1 is immobile at this timescale. At a longer timescale after FRAP, Knk1 levels were largely restored (over $60 \%$ compared with original levels) after varying time periods (Fig. S5K). However, once maximal Knk1 levels were reached, no plateau phase of the Knk1 intensity was detected. Instead, the Knk1 level dropped again, probably controlled by its oscillatory properties.

\section{Discussion}

We identified a novel component of fission morphogenesis, the $\mathrm{AAA}^{+}$ATPase Knk1, supporting linear growth in an MT/Tea1independent pathway.

Consistent with its role in morphogenesis, Knk1 localizes to cell tips and the septum region. Tip intensity is enhanced in the endocytic defective sla 2 mutant and in $c d c 42-L 160 S$ mutant cells that appear functional in early endocytic steps (31), independent of Sla2's role in endocytosis. In addition, double sla 2 cdc42 mutants mimicked single mutants, indicating that $\mathrm{Cdc} 42$ and Sla2 act in the same pathway. No direct link between these proteins has been reported in fission yeast, but in budding yeast the Cdc42 system is known to interact with the key endocytic proteinsepsins that function cooperatively with Sla2 to couple plasma membrane and actin cytoskeleton dynamics in early steps of endocytosis (35). Mutants in the epsin N-terminal homology (ENTH) domain displayed polarity defects resulting from decreased levels of active $\mathrm{Cdc} 42$, but endocytosis was unaffected. Because our study in fission yeast also suggests a link between the early endocytic adaptor protein Sla2 and Cdc42 in Knk1 localization, there may be a conserved connection between endocytic processes and the $\mathrm{Cdc} 42$ pathway linking endocytosis and cell polarization machinery to regulate downstream growth factors. Nevertheless, the exact mechanisms regulating Knk1 localization remain elusive at this point.

We detected an oscillatory behavior for Knk1 in sla2s and $c d c 42-L 160 S$ mutants that, at least in sla2s cells, does not seem to coincide with $\mathrm{Cdc} 42$ oscillations. Thus, our work suggests the coexistence of two oscillatory systems with distinct periodicities, for $\mathrm{Cdc} 42$ at $5 \mathrm{~min}$ and $\mathrm{Knk} 1$ at $15 \mathrm{~min}$. Future work will be required to test whether these systems are coupled. We did not detect clear periodic Knk1 fluctuations in wild-type cells. Here, cytoplasmic Knk1 intensity fluctuations are strong and might artifactually influence the analysis of the dynamics of tip intensities. Second, FRAP experiments suggest a fraction of highly dynamic Knk1 at cell tips, $30 \%$ in wild-type and $15 \%$ in sla $2 \Delta$ cells, whereas the turnover of more stably bound Knk1 molecules might be controlled by its oscillatory properties. This relatively large fraction of rapidly overturning Knk1 in wild-type cells may obscure its oscillatory signal. We therefore speculate that Knk1 oscillation also might occur at specific periods in wild-type cells. However, we cannot exclude the possibility that the periodic oscillations might be triggered by cellular alterations in sla $2 \Delta$ and cdc42-L160S, for example by limiting the amount of available Knk1 resulting in the competition of the two cell tips for Knk1. In fact, the enhanced Knk1 levels in sla2s and cdc42-L160S cells indicate that $\mathrm{Cdc} 42$ and Sla2 might be part of the negative feedback mechanism leading to cell tip detachment of Knk1. Under wild-type conditions, such striking anticorrelated periodic oscillations might not be observed; for example, regulatory proteins usually might be present in sufficient quantities, and thus the two cell tips would not compete for Knk1. Furthermore, it remains to be addressed whether Knk1's oscillatory behavior is required for its function in morphogenesis.

Interestingly, a simulation based on the Turing reaction-diffusion model, using MT-Tea1 reinforcement of growth zone and Cdc42 growth zone signaling, generated sharply bent fission yeast cells reminiscent of kinks (36). However, this model cannot be a mechanism for kink formation in the absence of Knk1, because $k n k 1 \Delta$ cells generate kinks independent of MT-Tea1.

The kinks seen in $k n k 1 \Delta$ cells are reminiscent of the behavior of fission yeast subjected to an electrical field, where cell growth is reoriented orthogonally to the field (37). This report shows 
that, in an electrical field, $\beta$-glucan synthases accumulate abnormally at one side of cell tips, leading to asymmetric cell wall synthesis. The knk1s phenotype might be caused by a similar asymmetrical distribution of certain polarity factors. However, no apparent cell wall abnormalities were observed with staining with the cell wall marker Calcolfluor (Fig. S6B). Interestingly, 1,3-beta-glucan synthase subunit 4 (Bgs4) (Fig. S6C), CRIB-GFP (Fig. S5F), and the actin cytoskeleton labeled by GFP-CDH (calponin homology domain) (Fig. S4B) in straight knk1s cells appeared undistinguishable from those in wild-type cells and also were symmetrically distributed along a kinked cell tip, suggesting that these components of the growth machinery have followed the new growth direction. Possibly, these components might be transiently relocalized during straight growth, resulting in kink formation. It remains elusive, whether Knk1 controls the distribution pattern of certain growth factors or whether Knk1 controls other processes at cell tips that in turn indirectly affect the localization of these proteins.

At this point, we can only speculate on how Knk1 may influence cell shape. $\mathrm{AAA}^{+}$ATPases are implicated in various cellular processes (17), e.g., membrane trafficking. However, we did not detect any endocytic (via FM4-64 uptake) or exocytic (via secretion of acid phosphatase; Fig. S6 $A$ ) defects, suggesting that $\mathrm{Knk} 1$ is not essential for these processes.

Alternatively, Knk1 might regulate the turnover of some celltip proteins via protein folding/unfolding, degradation, or protein complex disassembly, because many $\mathrm{AAA}^{+}$proteins are implicated in such processes (17). Potentially, by periodically removing components of the cell growth machinery, Knk1 might prevent the amplification of random deflection in growth

1. Mitchison JM, Nurse $P$ (1985) Growth in cell length in the fission yeast Schizosaccharomyces pombe. J Cell Sci 75:357-376.

2. Mishra M, Huang J, Balasubramanian MK (2014) The yeast actin cytoskeleton. FEMS Microbiol Rev 38(2):213-227.

3. Perez P, Rincón SA (2010) Rho GTPases: Regulation of cell polarity and growth in yeasts. Biochem J 426(3):243-253.

4. Das M, Wiley DJ, Chen X, Shah K, Verde F (2009) The conserved NDR kinase Orb6 controls polarized cell growth by spatial regulation of the small GTPase Cdc42. Curr Biol 19(15):1314-1319.

5. Martin SG, Rincón SA, Basu R, Pérez P, Chang F (2007) Regulation of the formin for $3 p$ by cdc42p and bud6p. Mol Biol Cell 18(10):4155-4167.

6. Iwaki T, Tanaka N, Takagi H, Giga-Hama Y, Takegawa K (2004) Characterization of end4+, a gene required for endocytosis in Schizosaccharomyces pombe. Yeast 21(10):867-881.

7. Castagnetti S, Behrens R, Nurse P (2005) End4/Sla2 is involved in establishment of a new growth zone in Schizosaccharomyces pombe. J Cell Sci 118(Pt 9):1843-1850.

8. Chang F, Martin SG (2009) Shaping fission yeast with microtubules. Cold Spring Harb Perspect Biol 1(1):a001347.

9. Huisman SM, Brunner D (2011) Cell polarity in fission yeast: A matter of confining positioning, and switching growth zones. Semin Cell Dev Biol 22(8):799-805.

10. Martin SG, McDonald WH, Yates JR, 3rd, Chang F (2005) Tea4p links microtubule plus ends with the formin for3p in the establishment of cell polarity. Dev Cell 8(4):479-491.

11. Mata J, Nurse P (1997) tea1 and the microtubular cytoskeleton are important for generating global spatial order within the fission yeast cell. Cell 89(6):939-949.

12. Brunner D, Nurse P (2000) CLIP170-like tip1p spatially organizes microtubular dynamics in fission yeast. Cell 102(5):695-704

13. Janson ME, Setty TG, Paoletti A, Tran PT (2005) Efficient formation of bipolar microtubule bundles requires microtubule-bound gamma-tubulin complexes. J Cell Biol 169(2):297-308.

14. Beinhauer JD, Hagan IM, Hegemann JH, Fleig U (1997) Mal3, the fission yeast homologue of the human APC-interacting protein EB-1 is required for microtubule in tegrity and the maintenance of cell form. J Cell Biol 139(3):717-728.

15. Busch KE, Brunner D (2004) The microtubule plus end-tracking proteins mal3p and tip 1p cooperate for cell-end targeting of interphase microtubules. Curr Bio/ 14(7):548-559.

16. Samejima I, Lourenço PC, Snaith HA, Sawin KE (2005) Fission yeast mto2p regulates microtubule nucleation by the centrosomin-related protein mto1p. Mol Biol Cell 16(6):3040-3051.

17. White SR, Lauring B (2007) AAA+ ATPases: Achieving diversity of function with conserved machinery. Traffic 8(12):1657-1667.

18. Kim DU, et al. (2010) Analysis of a genome-wide set of gene deletions in the fission yeast Schizosaccharomyces pombe. Nat Biotechnol 28(6):617-623.

19. Glynn JM, Lustig RJ, Berlin A, Chang F (2001) Role of bud6p and tea1p in the interaction between actin and microtubules for the establishment of cell polarity in fission yeast. Curr Biol 11(11):836-845

20. Unsworth A, Masuda H, Dhut S, Toda T (2008) Fission yeast kinesin-8 Klp5 and Klp6 are interdependent for mitotic nuclear retention and required for proper microtubule dynamics. Mol Biol Cell 19(12):5104-5115.

21. Hirota K, Tanaka K, Ohta K, Yamamoto M (2003) Gef1p and Scd1p, the Two GDP-GTP exchange factors for $\mathrm{Cdc} 42 \mathrm{p}$, form a ring structure that shrinks during cytokinesis in Schizosaccharomyces pombe. Mol Biol Cell 14(9):3617-3627. direction that otherwise is stabilized by positive feedback loops reinforcing the growth machinery locally (38).

Our study suggests that oscillatory properties represent a key feature in controlling cell shape. A first description of oscillatory dynamics emerged from studies in bacteria (39), where the MinCDE system undergoes oscillations to prevent division away from the cell middle. Remarkably, these oscillations are driven by the activity of the ATPase MinD. Future work may show that oscillatory dynamics are used commonly among ATPases or other NTPases to control various cellular processes.

\section{Experimental Procedures}

Standard fission yeast media and techniques were used as described (40). Live cell imaging was performed by spinning disk confocal microscopy as previously described (41). Frequency/periodicity of oscillations was obtained by FFT analysis using Matlab. Detailed descriptions are supplied in $S I E X-$ perimental Procedures. S. pombe strains used in this study are listed in Table S1. Knk1-2xGFP and CRIB-GFP dynamics in wild-type and mutant cells are quantified in Table $\mathbf{S 2}$.

ACKNOWLEDGMENTS. We thank the laboratories of J.-C. Ribas (University of Salamanca), P. Perez (University of Salamanca), and T. Pollard (Yale University) and the Japan National BioResource Project for generously providing reagents and Sergio Rincon for helpful technical advice and discussion. Imaging was performed at the Biolmaging Cell and Tissue Core Facility of the Institut Curie, a member of the France-Bioimaging National Research Infrastructure. K.S. is supported by a PhD fellowship from Complexité du Vivant-Université Pierre et Marie Curie and Fondation ARC pour la Recherche sur le Cancer. This work was supported by grants from the National Institutes of Health, the Agence Nationale de la Recherche, Institut National du Cancer, and Fondation ARC pour la Recherche sur le Cancer.

22. Hanson PI, Whiteheart SW (2005) AAA+ proteins: Have engine, will work. Nat Rev $\mathrm{Mol}$ Cell Biol 6(7):519-529.

23. Vajjhala PR, et al. (2008) The Vps4 C-terminal helix is a critical determinant for as sembly and ATPase activity and has elements conserved in other members of the meiotic clade of AAA ATPases. FEBS J 275(7):1427-1449.

24. Wendler $P$, Ciniawsky S, Kock M, Kube $S$ (2012) Structure and function of the AAA+ nucleotide binding pocket. Biochim Biophys Acta 1823(1):2-14.

25. Feierbach B, Chang F (2001) Roles of the fission yeast formin for $3 p$ in cell polarity, actin cable formation and symmetric cell division. Curr Biol 11(21):1656-1665.

26. Skruzny M, et al. (2012) Molecular basis for coupling the plasma membrane to the actin cytoskeleton during clathrin-mediated endocytosis. Proc Natl Acad Sci USA 109(38):E2533-E2542

27. Lee WL, Bezanilla M, Pollard TD (2000) Fission yeast myosin-I, Myo1p, stimulates actin assembly by Arp2/3 complex and shares functions with WASp. J Cell Bio/ 151(4):789-800.

28. Codlin S, Haines RL, Mole SE (2008) btn1 affects endocytosis, polarization of sterolrich membrane domains and polarized growth in Schizosaccharomyces pombe. Traffic 9(6):936-950

29. Sirotkin V, Beltzner CC, Marchand JB, Pollard TD (2005) Interactions of WASp, myosin-I, and verprolin with Arp2/3 complex during actin patch assembly in fission yeast. $J \mathrm{Ce} / \mathrm{H}$ Biol 170(4):637-648

30. Rincón SA, et al. (2009) Pob1 participates in the Cdc42 regulation of fission yeast actin cytoskeleton. Mol Biol Cell 20(20):4390-4399.

31. Estravís $M$, Rincón $S A$, Santos $B$, Pérez $P$ (2011) Cdc42 regulates multiple membrane traffic events in fission yeast. Traffic 12(12):1744-1758.

32. Estravis M, Rincon S, Pérez P (2012) Cdc42 regulation of polarized traffic in fission yeast. Commun Integr Biol 5(4):370-373.

33. Tatebe $\mathrm{H}$, Nakano K, Maximo R, Shiozaki K (2008) Pom1 DYRK regulates localization of the Rga4 GAP to ensure bipolar activation of Cdc42 in fission yeast. Curr Biol 18(5):322-330.

34. Das M, et al. (2012) Oscillatory dynamics of Cdc42 GTPase in the control of polarized growth. Science 337(6091):239-243.

35. Aguilar RC, et al. (2006) Epsin N-terminal homology domains perform an essential function regulating $\mathrm{Cdc} 42$ through binding $\mathrm{Cdc} 42$ GTPase-activating proteins. Proc Natl Acad Sci USA 103(11):4116-4121.

36. Drake T, Vavylonis D (2013) Model of fission yeast cell shape driven by membrane bound growth factors and the cytoskeleton. PLOS Comput Biol 9(10):e1003287.

37. Minc N, Chang F (2010) Electrical control of cell polarization in the fission yeast Schizosaccharomyces pombe. Curr Biol 20(8):710-716.

38. Slaughter BD, Smith SE, Li R (2009) Symmetry breaking in the life cycle of the budding yeast. Cold Spring Harb Perspect Biol 1(3):a003384.

39. Lutkenhaus J (2007) Assembly dynamics of the bacterial MinCDE system and spatial regulation of the $Z$ ring. Annu Rev Biochem 76:539-562.

40. Moreno S, Klar A, Nurse P (1991) Molecular genetic analysis of fission yeast Schizosaccharomyces pombe. Methods Enzymol 194:795-823.

41. Tran PT, Paoletti A, Chang F (2004) Imaging green fluorescent protein fusions in living fission yeast cells. Methods 33(3):220-225. 